\title{
Trap-Mediated Energy Transfer in Er-Doped Cesium Lead Halide Perovskite
}

Feng Jiang ${ }^{1 \#}$, Weihao Zheng ${ }^{2 \#}$, Ying Jiang ${ }^{1 \#,}$ Yang Li"\#, Peng Fan $^{1}$, Wei Huang ${ }^{1}$, Xianwei $F u^{2}$, Lihui Li1, Yu Ouyang ${ }^{1}$, Xiaoli Zhu', Xiujuan Zhuang ${ }^{1 *}$, Anlian Pan ${ }^{*}$

${ }^{1}$ Key Laboratory for Micro-Nano Physics and Technology of Hunan Province, School of Physics and Electronics, Hunan University, Changsha 410082, People's Republic of China

${ }^{2}$ Key Laboratory for Micro-Nano Physics and Technology of Hunan Province, College of Materials Science and Engineering, Hunan University, Changsha 410082, People's Republic of China

KEY WORDS: erbium-doped perovskites, shallow trap states, ions sensitization, trap-mediated energy transfer, time-resolved photoluminescence. 


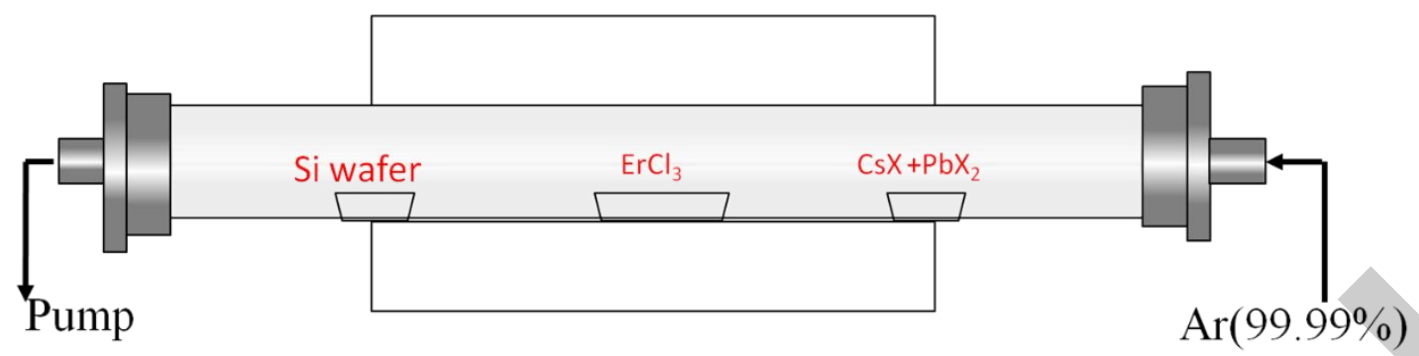

Figure S1. Set-up schematic for the growth of $\mathrm{Er}: \mathrm{CsPb}(\mathrm{Cl} / \mathrm{Br})_{3} \mathrm{MPs}$. By an in-situ source changing $\mathrm{CVD}$ route, the $\mathrm{Er}: \mathrm{CsPb}(\mathrm{Cl} / \mathrm{Br})_{3} \mathrm{MPs}$ can be grown up. An alumina boat containing of a mixture of $\mathrm{CsX}, \mathrm{PbX}_{2}(\mathrm{X}=\mathrm{Cl}, \mathrm{Br})$ and $\mathrm{ErCl}_{3}$ (Alfa Aesar, 99.99\%) powders was placed at the heating zone. All chemicals were purchased from Aladdin and used without any purification unless otherwise stated. Several pieces of Si substrate were placed at the downstream of the quartz tube to grow the samples. Similar methods are available for reference 1 and 2. Before the growth process, the high-purity Ar gas with high flow rate $(>500 \mathrm{sccm})$ was introduced into the system to clean the impurity gas. Then heated the furnace to $775^{\circ} \mathrm{C} \sim 785^{\circ} \mathrm{C}$ at high speed and keep the temperature for 5 10minutes. During this procedure, a constant flow of $35 \mathrm{SCCM}$ high-purity Ar gas was introduced as a carrier gas. During the growth process, the dopant related structural defects formed during growth of nanomaterials, which may further trigger the adsorption and then incorporation of dopants at high temperature that can give enough kinetics, it also can be explain by defect in $\mathrm{PVK}$ host $\left(\mathrm{Cl}^{-}\right.$vacancy and $\mathrm{Pb}^{2+}$ vacancy ) provide more channels for adsorption and then incorporation of dopant ions that play a positive role in enhancing the doping efficiency of the PVK. ${ }^{3}$ Continuous adsorption of foreign ions during the growth process that cause a rough surface and increased trap state density. In our experiment, we select the sample with rough surface and high dope concentration as research object. 


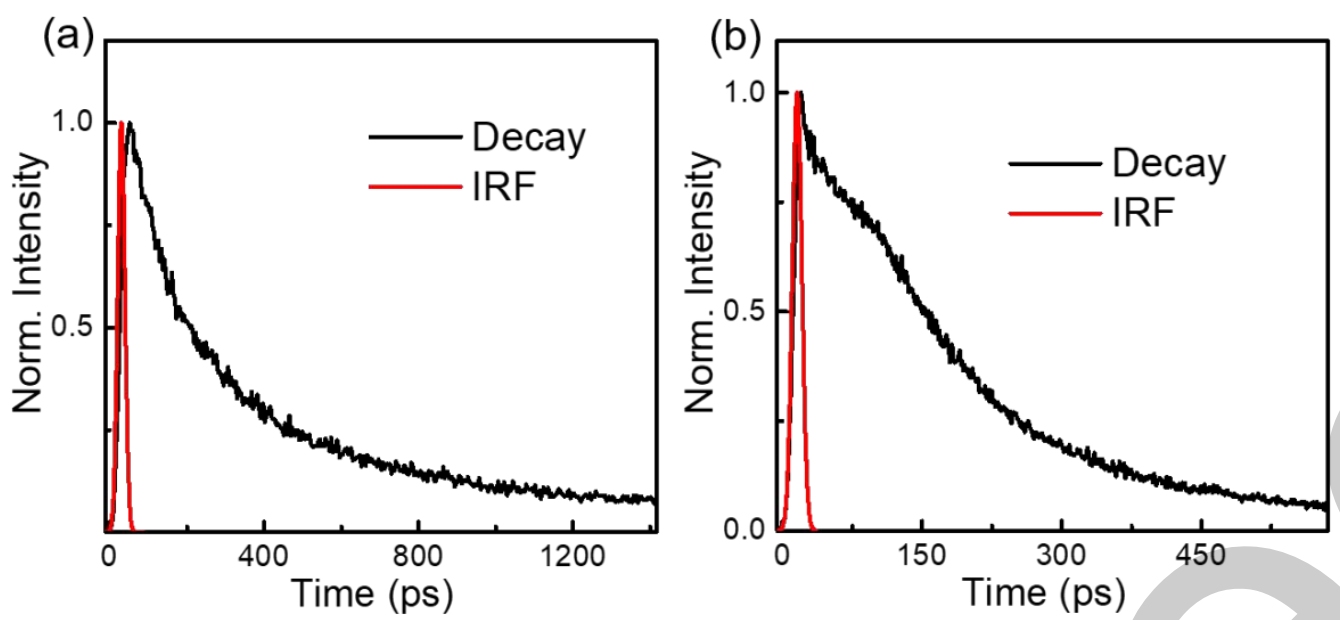

Figure S3. After normalizing the TRPL curve of PVK and the IRF curve. In undoped samples, we can obtain two components from the TRPL spectrum with different time scales through biexponential fitting: a dominant fast decay ( $\sim 147 \mathrm{ps})$ and a slow decay ( $\sim 0.92 \mathrm{~ns})$. This TRPL fit results follows instead a biexponential dynamic with a fast portion $\left(\tau_{1}\right)$ followed by a slower component $\left(\tau_{2}\right)$ can be explained considering the involvement of trap states, ${ }^{4}$ which lie very close to the excitonic state, in the trapping-detrapping process. Pérez-Prieto and coworkers have shown that in PVK films the photogenerated charge carriers or excitons migrate among samples, and during the migration, they collect into trap states, resulting in delayed emission. This behavior is typical of the copresence of direct exciton recombination (with lifetime $\tau_{1}$ ) and slower decay (with lifetime $\tau_{2}$ ) of regenerated excitons by detrap from non-emissive trap states that thermally repopulate the bandedge and provides a first important indication of the involvement of localized shallow states in the photo-physics of these samples. But for the doped sample, the lifetime exhibits more faster rate of its whole decay curve compared to that of undoped one and the effective value of the PVK carrier lifetime of $\sim 167 \mathrm{ps}$ and $\sim 351 \mathrm{ps}$ for doped and undoped respectively. 


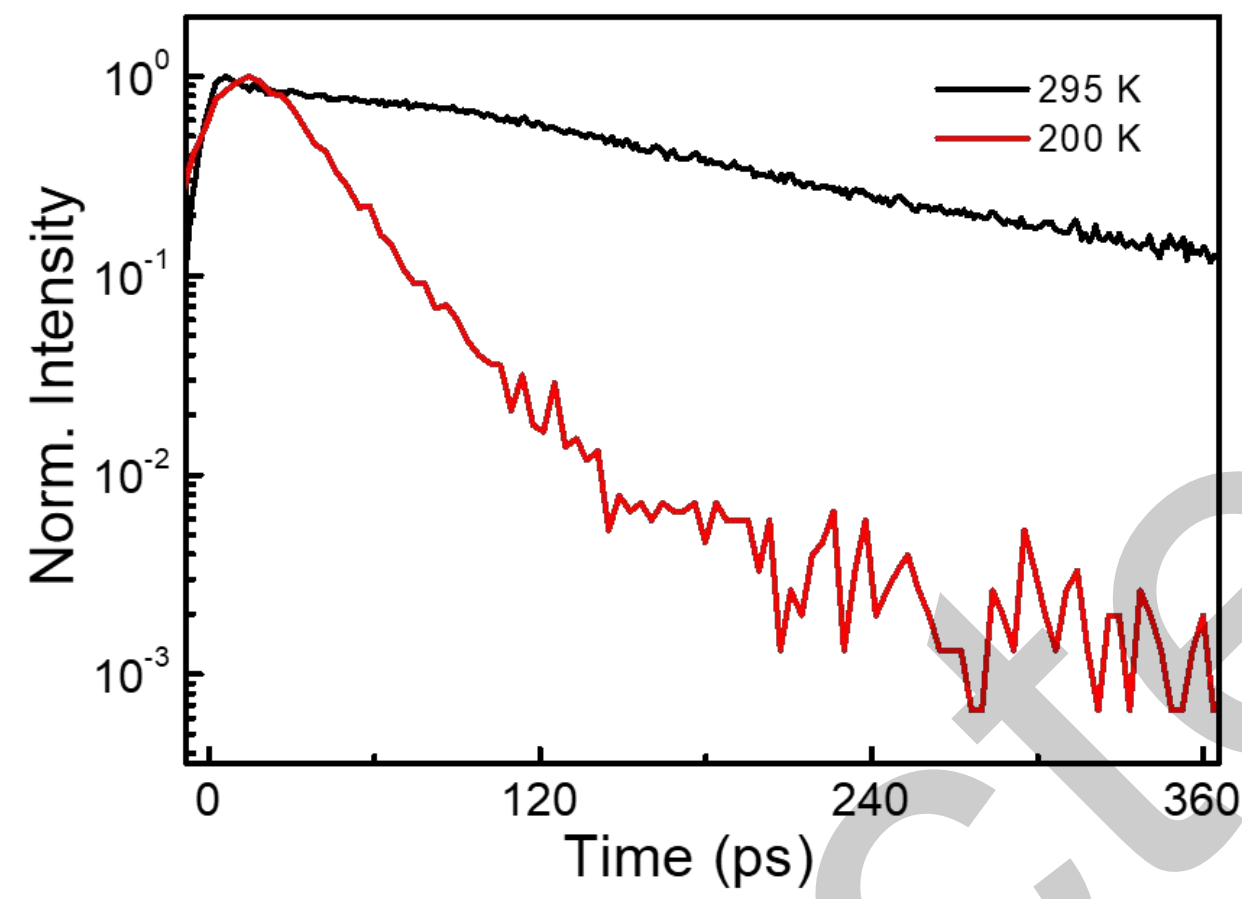

Figure S4. Transient photoluminescence spectra of PVK at 200 and 295 K. 


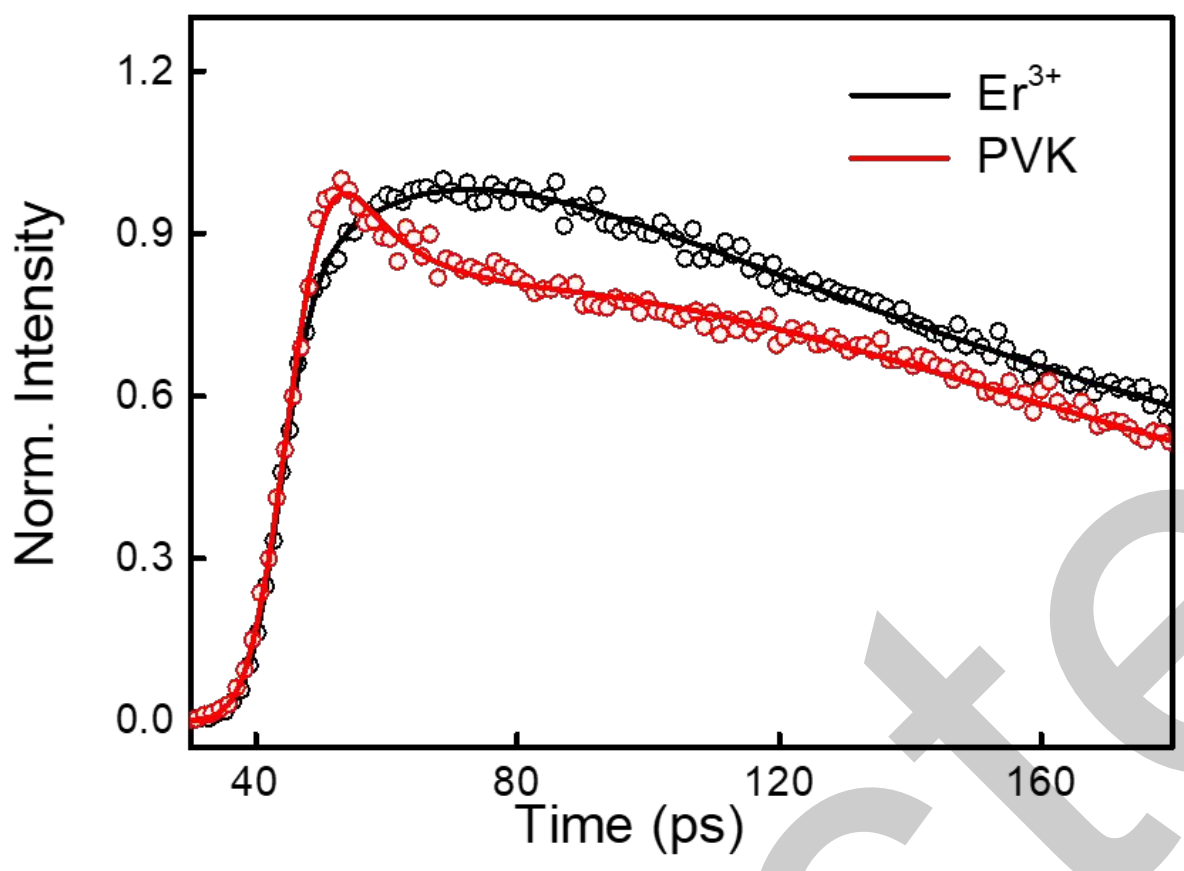

Figure S5. Decay curves taken from the $\mathrm{Er}^{3+}$ emission with the excitation power of 1.43 $\mu \mathrm{J} / \mathrm{cm}^{2}$.pulse. The rising time of the dynamic curves from $\mathrm{Er}^{3+}$ is determined to be 20 ps. 

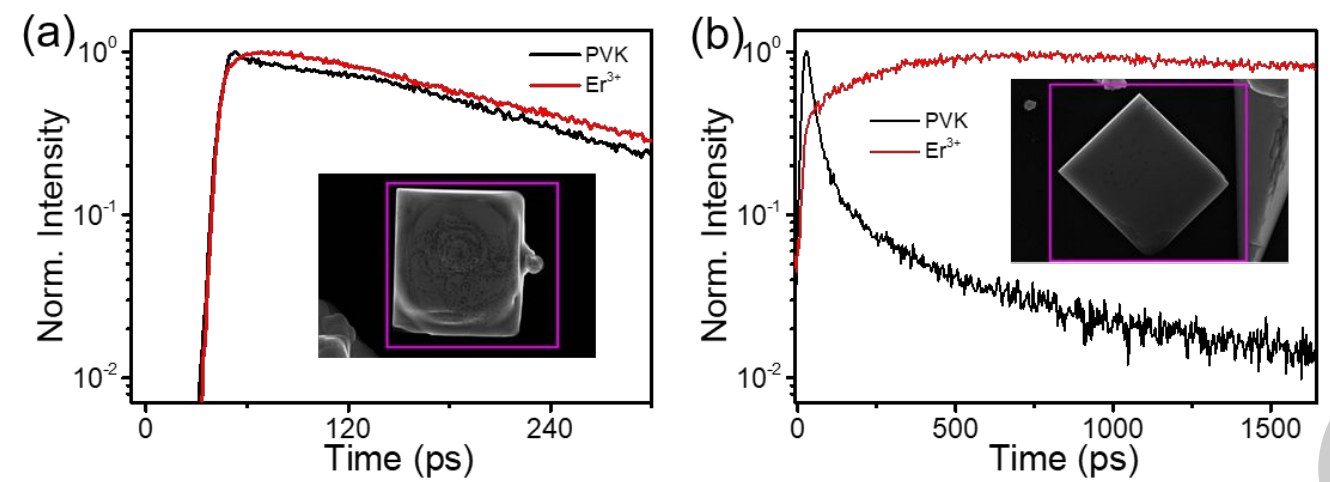

Figure S6. TRPL of both $\mathrm{Er}^{3+}$ and PVK in the sample (a) with worse shape and (b) regular shape. Insets respectively give the corresponding SEM images. The risetime are almost the same between the $\mathrm{Er}^{3+}$ and PVK of the sample in (a) with worse shape, indicating an efficient trap-mediated energy transfer and the abundant trap states. The risetime of $\mathrm{Er}^{3+}$ shows obvious longer than that of PVK of the sample in (b) with regular shape, indicating the direct energy transfer and the few amount of trap states. 


\section{Fitting details}

(a)
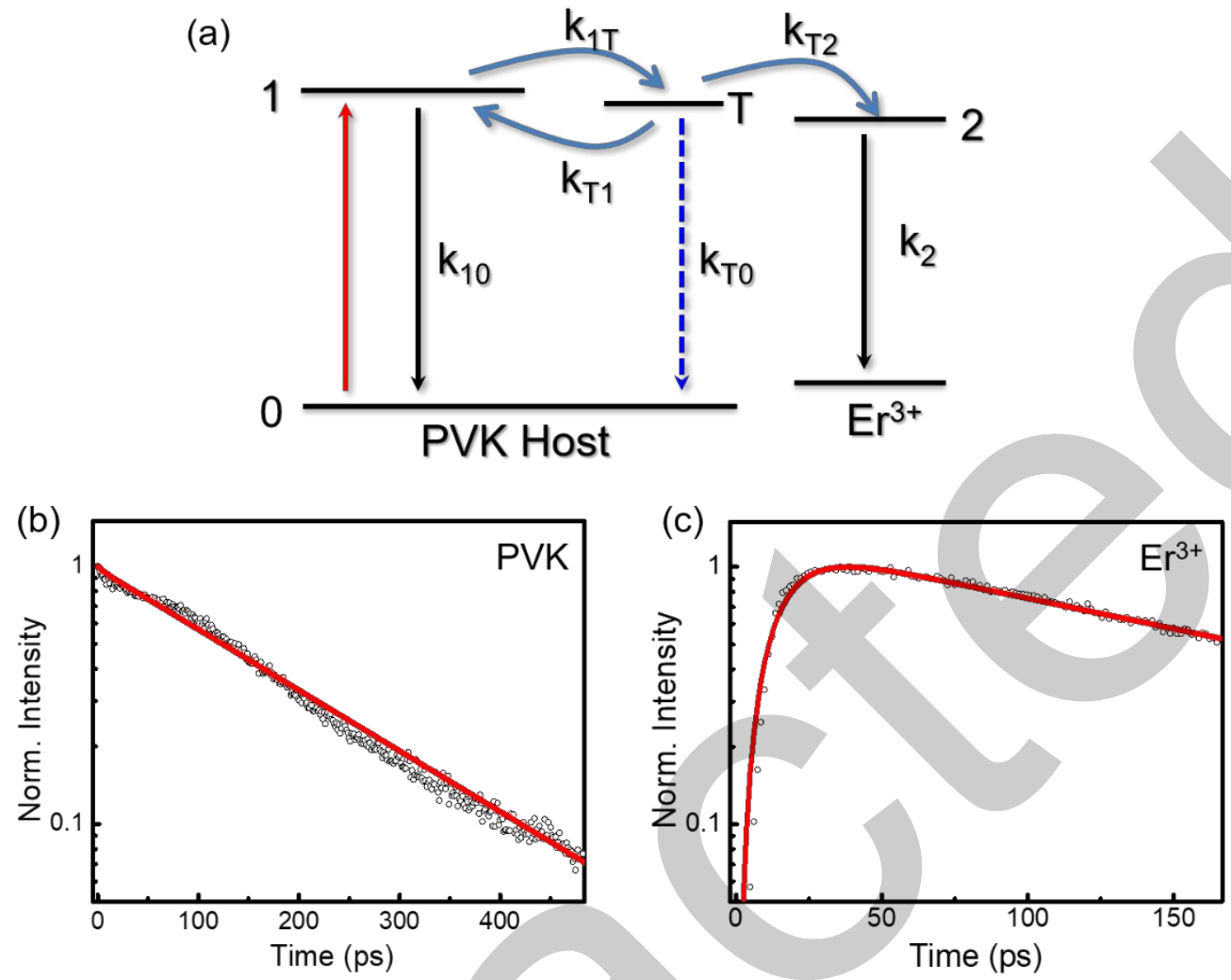

Figure S7. Energy diagram describing the ET mechanism with the trap state below the bottom of the conduction band. (a) The state 0 is the valence band edge, state 1 is the excitonic state, state 2 is the energy acceptor $\left(\mathrm{Er}^{3+}\right)$ and $\mathrm{T}$ is the trap state. The fast PL corresponds to the spontaneous radiative recombination of the excitons, with its rate constant $\mathrm{k}_{10}$. The delayed PL via the spontaneous recombination channel consequence of a multiple trapping-detrapping process. The rate constants $\mathrm{k}_{1 \mathrm{~T}}$ and $\mathrm{k}_{\mathrm{T} 1}$ describe the circular trapping-detrapping and define the relative population of the states and the nonradiative channel for the trap state deactivation is described by its rate constant $\mathrm{k}_{\mathrm{T} 0}$. The rate constants $\mathrm{k}_{\mathrm{T} 2}$ describe energy transfer rate and $\mathrm{k}_{2}$ describe radiative rate of energy acceptor $\left(\mathrm{Er}^{3+}\right) .(\mathrm{b})$ and (c) Dynamics of depopulation of the excitonic and $\mathrm{Er}^{3+}$ ions of Er-PVK MPs calculated by solving equations (2). 
Based on the labeled rates shown in the figure and the carrier population of each states, we present three kinetic equations as follow:

$$
\begin{aligned}
& \frac{\mathrm{d} N_{1}}{d t}=-\left(k_{10}+k_{1 T}\right) N_{1}+k_{T 1} N_{T} \\
& \frac{\mathrm{d} N_{T}}{d t}=k_{1 T} N_{1}-\left(k_{T 1}+k_{T 1}+k_{T 2}\right) N_{T} \\
& \frac{\mathrm{d} N_{2}}{d t}=k_{T 2} N_{T}-k_{2} N_{2}
\end{aligned}
$$

Equation (1), (2), and (3) respectively describe the carrier evolution of the excited state of PVK host, trap state, and the energy level of $\mathrm{Er}^{3+}$. Because that the excited state 1 and trap state $\mathrm{T}$ are connected with each other through a thermal activation mechanism with the photon playing an important role. The carrier trapping rate $\mathrm{K}_{1 \mathrm{~T}}$ and de-trapping rate $\mathrm{K}_{\mathrm{T} 1}$ obey the Maxwell-Boltzmann distribution and a relational expression can be defined as: $k_{T 1}=k_{1 T} \exp \left(-\Delta E / k_{B} T\right)$. ${ }^{4,7}$ Where $k_{B}$ is the Boltzmann constant, $T$ is the temperature, and $\Delta E$ is the energy difference between the 1 and the T state while this energy difference can largely influence the trapping and detrapping rates. The recombination rate $\mathrm{K}_{10}$ of the excited state of the PVK host can be determined by calculating the average lifetime of the decay curve in Figure 4a. In addition, the nonradiative recombination rate $\mathrm{K}_{\mathrm{T} 0}$ of the trap states can be ignored, since this recombination is indirect with the mismatch of its momentum space. Energy transfer rate $\mathrm{K}_{\mathrm{T} 2}$ can be determined by the difference of the risetime between the emission of the PVK host and $\mathrm{Er}^{3+}$ in the doped sample. Based on above relations and initial values, we then simulated the dynamic process of both PVK host and $\mathrm{Er}^{3+}$ as respectively shown in Figure S7b-c. The numerical solutions determined the energy difference $\Delta E$ with a value of $\sim-65 \mathrm{meV}$, the negative value imply that the trap states 
formed above the CBM, which mean that the electron trapping are difficult and therefore leading to an inefficient carrier trapping and de-trapping processes. 


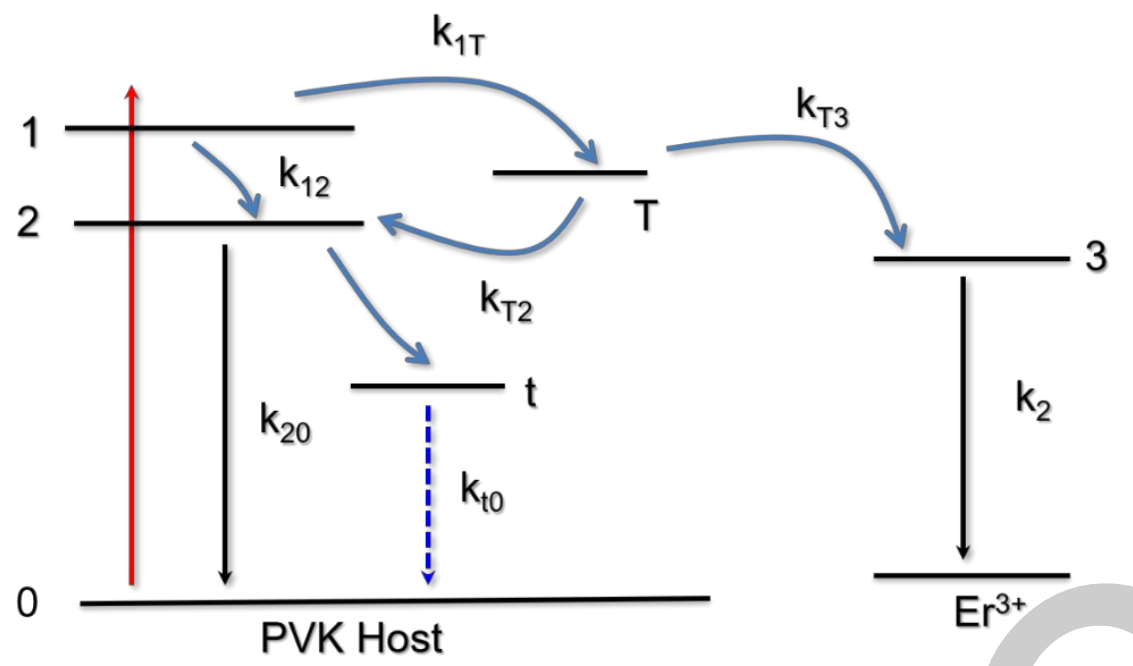

Figure S8. Energy diagram describing the energy transfer (ET) mechanism with the trap state above the bottom of the conduction band. The parameters used in simulation are $k_{12}=2000 \mathrm{ps}^{-1}, k_{1 T}=1000 \mathrm{ps}^{-1}$, and $k_{T 2}=50 \mathrm{ps}^{-1}$. The state 0 is the valence band edge, state 1 is the excited state, state 2 is the excitonic state, state 3 is the energy acceptor $\left(\mathrm{Er}^{3+}\right), \mathrm{T}$ is the trap state and $\mathrm{t}$ represent the nonradiative channels. The fast PL corresponds to the spontaneous radiative recombination of the excitons with its rate constant $\mathrm{k}_{10}$. The nonradiative channel for the deep trap state deactivation is described by its rate constant $\mathrm{k}_{\mathrm{t} 0}$. The rate constants $\mathrm{k}_{\mathrm{T} 3}$ describe energy transfer rate and $\mathrm{k}_{3}$ describe radiative rate of energy acceptor $\left(\mathrm{Er}^{3+}\right)$. 
Detail analysis about trap-mediated energy transfer: The trap state mediated the energy transfer can be verified and understand from follow points. Firstly, in the Er: $\mathrm{CsPb}(\mathrm{Cl} / \mathrm{Br})_{3} \mathrm{MPs}$, partial substitution of $\mathrm{Pb}^{2+}$ with $\mathrm{Er}^{3+}$ can reduce the extent of $\mathrm{Pb}$ X octahedral distortion, which will destroy the short-range order of the PVK lattice and produce trapping centers. The dopant related structural defects and heterovalent dopants can create impurity levels, either deep or shallow traps. If the dopant sensitization process through a direct pathway, the emission intensities of $\mathrm{Er}^{3+}$ can be described as $\mathrm{I}_{\mathrm{Er}} \propto \mathrm{k}_{\mathrm{ET}} \mathrm{N}_{2}-\mathrm{k}_{\mathrm{nr}} \mathrm{N}_{\mathrm{Er}}$. Second, as shown in the Figure S9, we observed that the emission intensity of the $\mathrm{Er}^{3+}$ is largely decreased at low temperature $(180 \mathrm{~K})$, which is consistent with the trap-mediated energy transfer in previous works. Normally, the process that the carrier captured by the trap states (process 5 in the Figure 4) should be limited at low temperature since the motion of the carrier is suppressed at that temperature. The decreased emission intensity of $\mathrm{Er}^{3+}$ at low temperature demonstrated again that the energy transfer process in our work experiences a trap-mediated process. Third, if the energy transfer to $\mathrm{Er}^{3+}$ proceeded by a direct pathway, the transfer time scale should be much slower with hundreds of picoseconds or even up to several nanoseconds as reported in previous works. ${ }^{8-10}$ In contrast, we only observed a very fast energy transfer process with the time scale of tens of picoseconds by analyzing the risetime of the decay curve in our experiments. This inconsistency suggests that energy transfer to $\mathrm{Er}^{3+}$ should occur via an alternative route rather than the normal excitondopant Dexter-type energy transfer. Take the above discussions into account, we trust that the energy transfer in our work mediated through the trap states. 


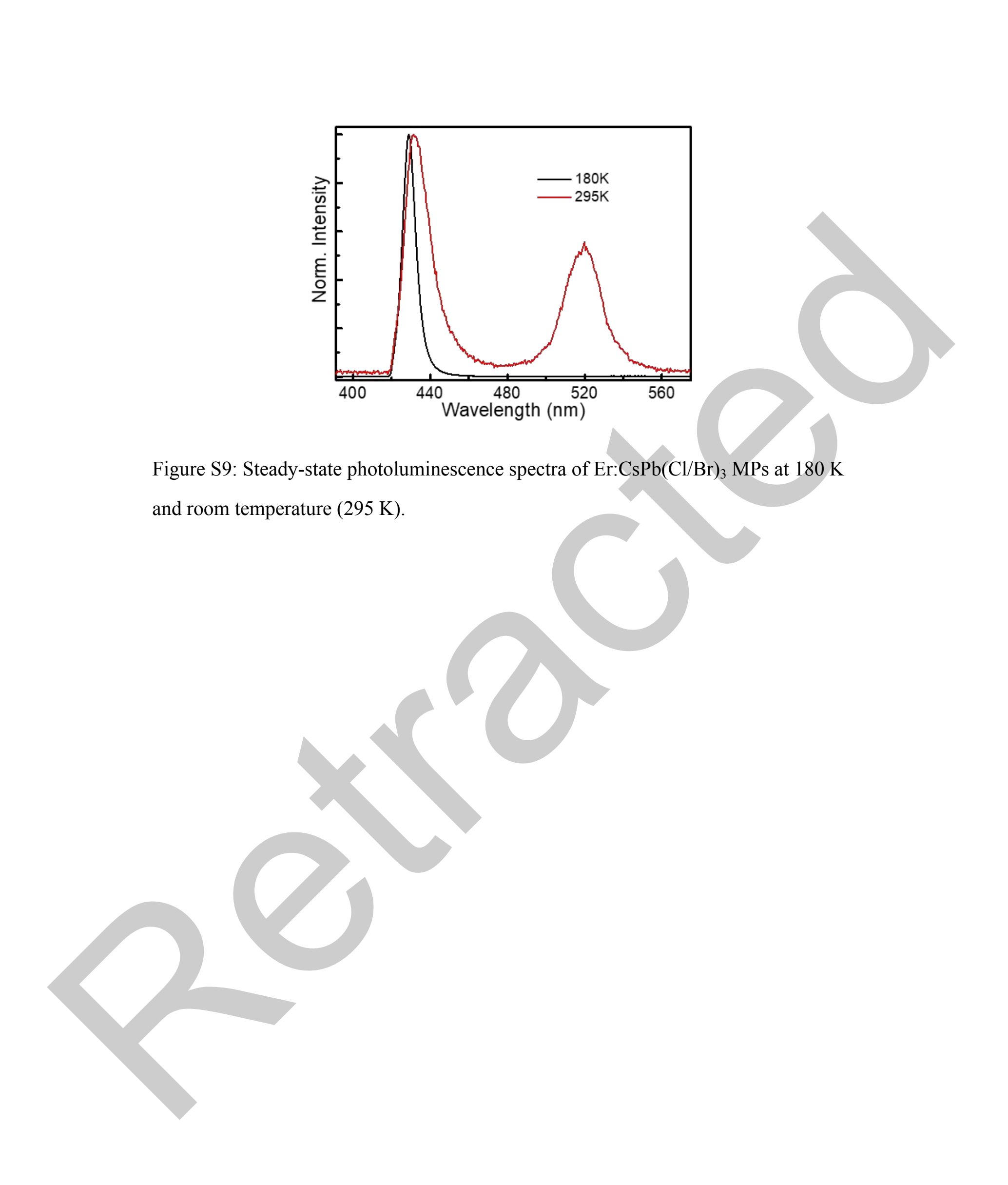


Table S1. EDS results of the sample.

\begin{tabular}{cll}
\hline Element & Weight(\%) & Atomic(\%) \\
\hline $\mathrm{Cl}$ & 19.02 & 50.07 \\
$\mathrm{Br}$ & 7.86 & 9.19 \\
$\mathrm{Cs}$ & 29.76 & 20.9 \\
$\mathrm{Er}$ & 2.81 & 1.57 \\
$\mathrm{~Pb}$ & 40.54 & 18.27 \\
\hline
\end{tabular}

Normally, in a pure $\mathrm{CsPbX}_{3} \mathrm{PVK}$, the $\mathrm{Cs}: \mathrm{Pb}: \mathrm{X}$ ratio is $1: 1: 3$. While in our Er-PVK MPs, we obtain the $\mathrm{Cs}:(\mathrm{Pb}+\mathrm{Er}):(\mathrm{Cl}+\mathrm{Br})$ ratio closer to $1: 1: 3$, which implies the Er tend to occupy the $\mathrm{Pb}$ site. 


\section{REFERENCE}

1.Hu, X.; Zhou, H.; Jiang, Z.; Wang, X.; Yuan, S.; Lan, J.; Fu, Y.; Zhang, X.; Zheng, W.; Wang, X.; Zhu, X.; Liao, L.; Xu, G.; Jin, S.; Pan, A., Direct Vapor Growth of Perovskite $\mathrm{CsPbBr}_{3}$ Nanoplate Electroluminescence Devices. ACS Nano 2017, 11, 9869-9876.

2.Zhou, H.; Yuan, S.; Wang, X.; Xu, T.; Wang, X.; Li, H.; Zheng, W.; Fan, P.; Li, Y.; Sun, L.; Pan, A., Vapor Growth and Tunable Lasing of Band Gap Engineered Cesium Lead Halide Perovskite Micro/Nanorods with Triangular Cross Section. ACS Nano 2017, 11, 1189-1195.

3.Ma, J.-P.; Chen, Y.-M.; Zhang, L.-M.; Guo, S.-Q.; Liu, J.-D.; Li, H.; Ye, B.-J.; Li, Z.Y.; Zhou, Y.; Zhang, B.-B.; Bakr, O. M.; Zhang, J.-Y.; Sun, H.-T., Insights into the local structure of dopants, doping efficiency, and luminescence properties of lanthanide-doped $\mathrm{CsPbCl3}$ perovskite nanocrystals. J. Mater. Chem. C 2019, 7 (10), 3037-3048.

4.Chirvony, V. S.; González-Carrero, S.; Suárez, I.; Galian, R. E.; Sessolo, M.; Bolink, H. J.; Martínez-Pastor, J. P.; Pérez-Prieto, J., Delayed Luminescence in Lead Halide Perovskite Nanocrystals. J. Phys. Chem. C 2017, 121, 13381-13390.

5.Xu, K.; Meijerink, A., Tuning Exciton-Mn ${ }^{2+}$ Energy Transfer in Mixed Halide Perovskite Nanocrystals. Chem. Mater. 2018, 30, 5346-5352.

6. Yuan, X.; Ji, S.; De Siena, M. C.; Fei, L.; Zhao, Z.; Wang, Y.; Li, H.; Zhao, J.; Gamelin, D. R., Photoluminescence Temperature Dependence, Dynamics, and Quantum Efficiencies in $\mathrm{Mn}^{2+}$-Doped $\mathrm{CsPbCl}_{3}$ Perovskite Nanocrystals with Varied Dopant Concentration. Chem. Mater. 2017, 29, 8003-8011.

7.Chen, L.; Li, B.; Zhang, C.; Huang, X.; Wang, X.; Xiao, M., Composition-Dependent Energy Splitting between Bright and Dark Excitons in Lead Halide Perovskite Nanocrystals. Nano Lett. 2018, 18, 2074-2080.

8.Milstein, T. J.; Kroupa, D. M.; Gamelin, D. R., Picosecond Quantum Cutting Generates Photoluminescence Quantum Yields Over 100\% in Ytterbium-Doped $\mathrm{CsPbCl}_{3}$ Nanocrystals. Nano Lett. 2018, 18, 3792-3799.

9.Pinchetti, V.; Anand, A.; Akkerman, Q. A.; Sciacca, D.; Lorenzon, M.; Meinardi, F.; Fanciulli, M.; Manna, L.; Brovelli, S., Trap-Mediated Two-Step Sensitization of Manganese Dopants in Perovskite Nanocrystals. ACS Energy Lett. 2018, 4, 85-93.

10.Parobek, D.; Roman, B. J.; Dong, Y.; Jin, H.; Lee, E.; Sheldon, M.; Son, D. H., Exciton-to-Dopant Energy Transfer in Mn-Doped Cesium Lead Halide Perovskite Nanocrystals. Nano Lett. 2016, 16, 7376-7380. 